\title{
EDITORIAL: \\ The ADHD challenge to practitioners
}

The national executive of the College, then based in the Victorial Section, organised a oneday scientific retreat on attention deficit hyperactivity disorder in September 1995. The retreat took advantage of the presence in Australia of ADHD researcher Russell Barkley for a CEDP workshop tour of Perth and Melbourne. Practitioners and researchers from several disciplines and from several states were given the opportunity to meet, share their work, and attempt to articulate a basic consensus about ADHD, its developmental nature, and its educational consequences.

This special double issue arises from that retreat. The November 1995 issue of AEDP was cancelled and incorporated into this May 1996 issue. This issue provides a resource for members of the college that represents some of the most visible research and practice in relation to ADHD. Identification and measurement of ADHD and formulation of the nature of an individual child's behaviour are difficult tasks that are the subject of widespread efforts at this time.

For this issue, Russell Barkley has outlined his emerging theory, to be published in more detail in Psychological Bulletin, and has provided a general perspective on the field. Psychologists Margot Prior and Matt Sanders, who direct major Australian research programs on children's development, have reviewed their particular perspectives on ADHD in relation to learning difficulties and behaviour problems, respectively. David Mellor and his colleagues have articulated the demands-resources dilemna facing service providers in meeting perceived needs of families. Pediatricians Rick Jarman and Trevor Parry have reviewed management options and the need for individualised treatment strategies. Neurologist Richard Silberstein has outlined experimental work using the Continuous Performance Task that links AHDH to planning. Behaviour geneticist David Hay has provided data on the origin and dimensional nature of ADHD and its impact on interpretation of categorical diagnosis. developmental problems having substantial community impact. It is becoming increasingly substantial, rich, and useful. Postgraduate research in progress is contributing more effort in addressing to this very human challenge to our society. The NH and MRC is presently considering research funding for more medical and epidemiological work on ADHD. Measurement issues of item relevance, task criteria, and subtypes leave many tantalising psychological tasks to be pursued.

The process of reaching for consensus at the end of the retreat was conservative and cautious in nature, particularly on the central debates of drugs and behavioural treatment. Russell Barkley had argued strongly for the chronic nature of ADHD as a developmental disorder, requiring drug as well as behavioural management. Issues of early screening for behavioural problems and comorbid influence of oppositional and conduct disorder and other psychopathologies on long-term outcomes were tangled with this discussion. Barkley also argued strongly that the disorder is behavioural rather than attentional, and yet several papers in this issue demonstrate that the attentional manifestations of educational difficulties needs to be analysed and examined carefully.

Members of the college are invited to contribute practitioner responses about their continuing concerns and needs and theoretical commentaries. As AEDP editorial policy now seeks to promote exemplary practice, case reports are also of interest.

Members of the college will find much on which to reflect in this issue. Problems remain of Australian prevalence and severity, state-bystate attitude to medication, and treatment: who needs treatment, who treats, with what approach, where does the treatment take place, and how treatments coordinate service providers in an effective manner. 